\title{
LOCALLY NONCOMMUTATIVE SPACETIMES
}

\author{
JAKOB G. HELLER, NIKOLAI NEUMAIER AND STEFAN WALDMANN
}

Communicated by Martin Schlichenmaier

\begin{abstract}
A rather new physically well-motivated notion of locally noncommutative spacetimes - a refinement of the notion noncommutative spacetimes - is reviewed and discussed. It is shown that the latter model can be realized in the framework of formal deformation quantization using star products as well as in the framework of Rieffel's strict deformation quantization. For the convergent setting Rieffel's former results for $C^{*}$-algebras are generalized to pro- $C^{*}$-algebras and applied to actions well-suited to the idea of locally noncommutative spacetimes.
\end{abstract}

\section{Introduction and Motivation}

Since the simultaneous validity of quantum theory and general relativity has to break down at the Planck scale, various models for spacetime geometry at such scales have been discussed over the years. One promising approach is to deform the classical commutative geometry of spacetime into a noncommutative geometry and study dynamics like (quantum) field theories on this noncommutative spacetime, see e.g., the pioneering work [3]. Here many versions have been discussed, though all of them have one feature in common: the noncommutativity is global and hence has global consequences. This is reflected in the famous UV/IR mixing in the Euclidian versions of field theory and in rather absurd dispersion relations in the truly Minkowski versions, see e.g., $[1,8]$ and references therein.

One particular example which has been studied in detail is the noncommutative Minkowski spacetime with the usual Weyl-Moyal star product quantizing a constant Poisson structure. Mathematically speaking, this is equivalent to the quantization of a non-relativistic particle with two-dimensional configuration space $\mathbb{R}^{2}$ and hence phase space $\mathbb{R}^{4}$. Though quantum mechanics only appears at small distances in configuration space, in phase space quantum mechanical effects are visible globally. This simple heuristic consideration already gives a hint why the much more subtle effects of UV/IR mixing etc. have to be expected. The reason is that the geometry of spacetime is globally noncommutative. 
However, the classical geometry is not wrong by itself, at least at scales we are confronted with in our daily life. Only at very small distances the notions of classical geometry lose their meanings. Thus we advocate for a refined approach to noncommutative spacetimes where the noncommutativity only appears for small distances. The idea is very simple: it is not the spacetime manifold $M$ which should be endowed with a noncommtutative structure but $M \times M$ as distance is a concept referring to two points.

The general framework will be to use a Poisson structure on $M \times M$ whose support is close to the diagonal $\Delta_{M}$ and study quantizations of this Poisson structure. One crucial requirement will be that the Poisson structure and the corresponding star product will be only nontrivial for functions which are sensitive to relative coordinates on $M \times M$. The model we will discuss comes in two flavours, formal and strict. While for the formal star products there is no restriction on the Poisson structure, the strict deformation will require Poisson structures which arise from an action of $\mathbb{R}^{d}$. In this case we can rely on Rieffel's deformation [11].

The present paper is based on a joint work with Bahns [2] as well as on [5]. Here we shall review and discuss both approaches and their relations and point out some of their features.

The paper is organized as follows: In Section 2 we make our idea of locally noncommutative spacetimes more precise. Section 3 gives the positive answer to the question of existence and classification of the latter in the framework of formal deformation quantization. In order to present the convergent counterpart of the existence result, we recall the basics of Rieffel's strict deformation quantization for $C^{*}$-algebras in Section 4 which we generalize to pro- $C^{*}$-algebras in Section 5. In the subsequent section we apply Rieffel's construction using actions with compact support. With the aid of these results we finally manage to give non-perturbative counterparts in the framework of (pro-) $C^{*}$-algebras of the noncommutative spacetimes, in Section 7.

\section{Locally Noncommutative Spacetimes}

Let us now recall the basic construction of locally noncommutative spacetimes from $[2,5]$. Our aim is to provide a framework for noncommutative spacetimes such that

- it works on generic spacetimes $(M, g)$

- it has a truly geometric formulation 
- it uses either formal or strict deformation quantization

- it modifies geometry only at small distances

in order to overcome the problems arising from models with global noncommutativities as discussed in the introduction. In this section, we focus on the formal deformation quantization case, though parts of the construction will still be valid for the strict case as well.

The last requirement immediately gives the correct hint how to proceed. Since distance is a concept involving two points one has to impose noncommutativity of $M \times M$ and not of $M$ alone: we want $M \times M$ to become noncommutative close to the diagonal $\Delta_{M} \subseteq M \times M$ but keep ordinary geometry far away from $\Delta_{M}$.

To formulate things properly, we use some geometric pre-requisites present on any (pseudo-)Riemannian manifold. In fact, we only need a torsion-free connection $\nabla$ on $M$ which typically (but not necessarily) will be the Levi-Civita connection of the metric $g$. We denote by $\pi: T M \longrightarrow M$ the tangent bundle of $M$ with zero section $\iota: M \longrightarrow T M$. One chooses an open neighbourhood $\mathcal{U} \subseteq T M$ of the zero section such that the map

$$
\Phi: \mathcal{U} \ni v_{p} \mapsto \Phi\left(v_{p}\right)=\left(\exp _{p}\left(-v_{p}\right), \exp _{p}\left(v_{p}\right)\right) \in \mathcal{V} \subseteq M \times M
$$

is a diffeomorphism onto its image $\mathcal{V} \subseteq M \times M$, where $\mathcal{V}$ is an open neighbourhood of the diagonal $\Delta_{M}$. Here and in the following, $\exp _{p}$ is the exponential map of $\nabla$. Clearly, such a choice of $\mathcal{U}$ can be achieved. The idea is then that the functions $C^{\infty}(M \times M)$ play the role of observables when it comes to questions involving two points. In particular, for points $q, q^{\prime}$ sufficiently close such that $\left(q, q^{\prime}\right) \in \mathcal{V}$, their geodesic midpoint $p \in M$ is uniquely defined by $p=\pi\left(\Phi^{-1}\left(q, q^{\prime}\right)\right)$.

Next, one chooses a Poisson structure $\tilde{\theta} \in \Gamma^{\infty}\left(\Lambda^{2} T(M \times M)\right)$ whose support is contained in $\mathcal{V}$. The idea is that $\mathcal{V}$ is still large compared to the region of noncommutativity $\operatorname{supp} \tilde{\theta}$. In particular, the precise choice of $\mathcal{V}$ should not play a major role, as we are interested in much smaller neighbourhoods of the diagonal $\Delta_{M}$. By $\Phi$ we can pull-back $\tilde{\theta}$ and obtain a Poisson structure $\theta=\Phi^{*} \tilde{\theta} \in \Gamma^{\infty}\left(\Lambda^{2} T(T M)\right)$ on $T M$ with support in $\mathcal{U}$.

Next, we require that for each $p \in M$ the set $\operatorname{supp} \theta \cap T_{p} M$ is a compact subset of $T_{p} M$ : this way, the range of noncommutativity is encoded to be small by using a topological characterization.

Having such a Poisson structure $\theta$ on $T M$ or, equivalently, $\tilde{\theta}$ on $M \times M$, we endow $T M$ with a star product $\star$ quantizing $\theta$. Equivalently, we obtain via $\Phi^{*}$ a 
star product $\tilde{\star}$ on $M \times M$. Since $\star$ and $\tilde{\star}$ encode the noncommutativity beyond the semiclassical level (which is $\theta$ and $\tilde{\theta}$, respectively), we require the same support conditions: outside $\operatorname{supp} \theta$, the star product $\star$ has to be the pointwise product and similarly for $\tilde{\star}$.

Up to now the model is not yet very specific as $\theta$ and hence $\star$ are still quite arbitrary and the support condition is not really a strong requirement. One suggestion to make the whole approach more interesting is now the following verticality requirement: we do not only want noncommutativity to show up at small distances but we even require that small distances are the only reason for noncommutativity. Thus we want an observable $f \in C^{\infty}(M \times M)$ to behave entirely classically if it is not sensitive to relative distances. Note that in general on $M \times M$ there is no intrinsic notion of relative coordinates. However, since we have a connection we can use the map $\Phi$ to induce relative coordinates as those directions transversal to $\Delta_{M}$ at $p$ which come under $\Phi$ from those directions of $T M$ along the tangent space $T_{p} M$. Thus we require: if $\left.f\right|_{\mathcal{V}}$ is constant along these relative coordinates, then for all other functions $g \in C^{\infty}(M \times M)$

$$
\{f, g\}_{\tilde{\theta}}=0 \quad \text { and also } \quad f \tilde{\star} g=f g=g \tilde{\star} f .
$$

The second requirement is just the quantized version of the semi-classical first statement. Translating these requirements back to $T M$ via $\Phi^{*}$ gives the equivalent conditions

$$
\left\{\pi^{*} u, g\right\}_{\theta}=0 \quad \text { and also } \quad \pi^{*} u \star g=\pi^{*} u g=g \star \pi^{*} u
$$

for all functions $u \in C^{\infty}(M)$ and $g \in C^{\infty}(T M)$, since a function $f$ which is constant along the fibers is of the form $\pi^{*} u$. The condition (3) explains the name 'vertical' since a Poisson tensor $\theta$ satisfies (3) iff $\theta \in \Gamma^{\infty}\left(\Lambda^{2} \operatorname{Ver}(T M)\right)$ where $\operatorname{Ver}(T M)=\operatorname{ker} T \pi \subseteq T(T M)$ is the vertical subbundle. Moreover, $\star$ satisfies (3) iff the cochains of $\star$ differentiate only in vertical directions.

To illustrate this condition we shall also give the local description in coordinates. Choose a local coordinate system $(U, x)$ on $M$. Then we denote the induced bundle coordinates of $T M$ by $(T U,(q, v))$ where $T U=\pi^{-1}(U) \subseteq T M$ is open and $q^{i}=\pi^{*} x^{i}$ while $v^{i}\left(v_{p}\right)=\mathrm{d} x^{i}\left(v_{p}\right)$ for $i=1, \ldots, n=\operatorname{dim}(M)$. Then $\star$ is written as

$$
f \star g=\sum_{r=0}^{\infty} \lambda^{r} C_{r}(f, g)
$$


with bidifferential operators $C_{r}: C^{\infty}(T M) \times C^{\infty}(T M) \longrightarrow C^{\infty}(T M)$. The verticality implies that locally with multi-index notation

$$
\left.C_{r}(f, g)\right|_{T U}=\sum_{I, J} C_{r}^{I J} \frac{\partial^{|I|} f}{\partial v^{I}} \frac{\partial^{|J|} g}{\partial v^{J}}
$$

and locally defined functions $C_{r}^{I J} \in C^{\infty}(T U)$. The support condition on $\star$ is equivalent to the condition that $C_{r}^{I J}$ has compact support in fiber direction and $\operatorname{supp} C_{r}^{I J} \subseteq T U \cap \mathcal{U}$.

Before we come to the existence and classification results we point out several consequences and properties of the model.

First we note that thanks to the verticality we can restrict $\theta$ as well as $\star$ to each tangent space. Denoting by $\iota_{p}: T_{p} M \longrightarrow T M$ the inclusion map we thereby obtain for each $p \in M$ a well-defined bivector field $\theta_{p} \in \Gamma^{\infty}\left(\Lambda^{2} T\left(T_{p} M\right)\right)$ on $T_{p} M$ such that $\theta_{p}$ and $\theta$ are $\iota_{p}$-related. In particular, $\theta_{p}$ is a Poisson structure itself and $\iota_{p}$ is a Poisson map

$$
\iota_{p}^{*}\left(\{f, g\}_{\theta}\right)=\left\{\iota_{p}^{*} f, \iota_{p}^{*} g\right\}_{\theta_{p}} .
$$

Analogously, $\star$ restricts to an associative star product $\star_{p}$ on $T_{p} M$ which quantizes $\theta_{p}$ such that $\iota_{p}^{*}$ becomes an algebra homomorphism

$$
\iota_{p}^{*}(f \star g)=\left(\iota_{p}^{*} f\right) \star_{p}\left(\iota_{p}^{*} g\right)
$$

The physical interpretation of this product $\star_{p}$ comes from the original picture of $M \times M$. If we are interested in observables related to events taking place at $q$ and $q^{\prime}$ then only the functions sensitive to their relative coordinates play a role. They can be obtained from $C^{\infty}\left(T_{p} M\right)$ where $p$ is the geodesic midpoint of $q$ and $q^{\prime}$. So for this situation only the algebra $\left(C^{\infty}\left(T_{p} M\right)[[\lambda]], \star_{p}\right)$ is relevant. Note that if $q$ and $q^{\prime}$ are not within $\mathcal{V}$ such that $p$ is well-defined, they are far apart, so no noncommutativity is to be expected anyway.

We can even interpret the situation as follows. Since $\theta_{p}$ and also $\star_{p}$ are only nontrivial inside $\mathcal{U}_{p}=\mathcal{U} \cap T_{p} M$ which is an open neighbourhood of $0_{p} \in T_{p} M$ we can push forward everything with $\exp _{p}$. Indeed, on $\mathcal{U}_{p}$ the exponential map $\exp _{p}$ is still a diffeomorphism onto its image $\mathcal{V}_{p}=\exp _{p}\left(\mathcal{U}_{p}\right) \subseteq M$ which is an open neighbourhood of $p \in M$. Thus we obtain a Poisson structure $\tilde{\theta}_{p}$ together with a star product $\tilde{\star}_{p}$ on $M$ which are non-trivial only inside the neighbourhood $\mathcal{V}_{p}$ of $p$. Again, the interpretation comes from the original $M \times M$ picture: whenever $p$ is the geodesic midpoint of $q, q^{\prime}$ and we are interested in physical 
processes involving the two points $q$ and $q^{\prime}$ then we have to use the observable algebra $\left(C^{\infty}(M)[[\lambda]], \tilde{\star}_{p}\right)$. In this way, every point in $M$ obtains its own small noncommutative neighbourhood since $\tilde{\star}_{p}$ is commutative outside of $\mathcal{V}_{p}$. This last observation is the motivation to call the spacetime locally noncommutative.

\section{Existence and Classification in the Formal Case}

Let us now discuss whether we actually can find vertical Poisson structures and corresponding star products meeting all our previous requirements. We start with the following construction:

Lemma 1. There exists a diffeomorphism $\Psi: B_{1}(0) \longrightarrow \mathbb{R}^{n}$ with

1. $\Psi$ is the identity on $B_{\frac{1}{2}}(0)$

2. The vector field $X$ with $X_{i}(p)=\left(\Psi^{*} \frac{\partial}{\partial x^{i}}\right)(p)$ for $p \in B_{1}(0)$ and $X_{i}(p)=0$ for $p \in \mathbb{R}^{n} \backslash B_{1}(0)$ is smooth on $\mathbb{R}^{n}$

3. $\Psi$ is $\mathrm{O}(n)$-invariant.

Since this diffeomorphism is $\mathrm{O}(n)$-invariant, it is well-defined on any vector bundle $\pi: E \longrightarrow M$ with fiber metric $h$. Thus we have a diffeomorphism $\Psi: B_{1}^{h}(0) \subseteq E \longrightarrow E$ with $\pi \circ \Psi=\pi$. If $e \in \Gamma^{\infty}(E)$ is a section, we denote by $e^{\text {ver }} \in \Gamma^{\infty}(\operatorname{Ver}(E)) \subseteq \Gamma^{\infty}(T E)$ its vertical lift. Analogously, we have vertical lifts for $X \in \Gamma^{\infty}\left(\bigotimes^{\bullet} E\right)$ to $X^{\text {ver }} \in \Gamma^{\infty}\left(\bigotimes^{\bullet} \operatorname{Ver}(E)\right)$.

Proposition 2. Let $\pi: E \longrightarrow M$ be a vector bundle with fiber metric $h$ and $\Psi$ as above.

1. For any $X \in \Gamma^{\infty}\left(\otimes^{\bullet} \operatorname{Ver}(E)\right)$ the tensor field $\Phi^{*} X^{\mathrm{ver}}$ is a globally defined, smooth tensor field on $E$ with support in $B_{1}^{h}(0)$ which coincides with $X^{\mathrm{ver}}$ on $B_{\frac{1}{2}}^{h}(0)$

2. For any $\gamma \in \Gamma^{\infty}\left(\Lambda^{2} E\right)$ the vertical lift $\gamma^{\text {ver }} \in \Gamma^{\infty}\left(\Lambda^{2} T E\right)$ is a vertical Poisson structure which is constant along the fibers

3. $\theta=\Psi^{*} \gamma^{\text {ver }} \in \Gamma^{\infty}\left(\Lambda^{2} T E\right)$ is a vertical Poisson structure with $\operatorname{supp} \theta \subseteq$ $B_{1}^{h}(0)$

4. There exists a number $d$, constants $\Theta^{i j}=-\Theta^{j i} \in \mathbb{R}$ and commuting vertical vector fields $X_{i} \in \Gamma^{\infty}(T E)$ with $\operatorname{supp} X_{i} \subseteq B_{1}^{h}(0)$ for $i, j=1, \ldots, d$ such that

$$
\theta=\frac{1}{2} \sum_{i, j} \Theta^{i j} X_{i} \wedge X_{j}
$$


Proof: The first three parts are clear by Lemma 1 as vertical lifts always commute with respect to the Schouten-bracket. The last part uses the Serre-Swan theorem, see [5, Proposition 2.3].

Note that each vector field $X_{i}$ has a complete flow as it is vertical and in vertical direction the support is compact thanks to supp $X_{i} \subseteq B_{1}^{h}(0)$. In particular, their flows constitute a smooth action of $\mathbb{R}^{d}$. This motivates the following definition: let $\mathcal{U}$ be an open neighbourhood of the zero section of $E$. Then a vertical Poisson structure $\theta$ on $E$ is called $\mathcal{U}$-admissible, if there exists a smooth action of $\mathbb{R}^{d}$ on $E$ by vertical diffeomorphisms such that the fundamental vector fields $X_{i}$ of this action have compact support in fiber directions and $\theta$ can be written as in (8). Then the last part of the proposition states that for any $\mathcal{U}$ we have a lot of $\mathcal{U}$-admissible vertical Poisson structures since we always can find a fiber metric $h$ such that $B_{1}^{h}(0) \subseteq \mathcal{U}$.

Coming back to the locally noncommutative spacetime, we consider $E=T M$ and conclude that there always exist many Poisson structures meeting all our requirements discussed in Section 2. We may even choose them to be $\mathcal{U}$-admissible.

Now we turn to the existence of star products $\star$ quantizing such a vertical Poisson structure. Here the situation is governed by Kontsevich's formality theorem on $\mathbb{R}^{n}$. We shall not go into the technical details of this theorem but outline some of its consequences.

When discussing formal star products, it is also convenient to consider formal Poisson structures

$$
\theta=\theta_{0}+\lambda \theta_{1}+\cdots=\sum_{r=0}^{\infty} \lambda^{r} \theta_{r} \in \Gamma^{\infty}\left(\Lambda^{2} T(T M)\right)[[\lambda]]
$$

where the Jacobi identity $\llbracket \theta, \theta \rrbracket=0$ has to be fulfilled order by order in the formal parameter $\lambda$. In particular, $\llbracket \theta_{0}, \theta_{0} \rrbracket=0$ and $\llbracket \theta_{0}, \theta_{1} \rrbracket=0$, whence $\theta_{0}$ is a Poisson structure itself and $\theta_{1}$ is a cocycle in the Poisson cohomology with respect to $\theta_{0}$. The relevant moduli space is then obtained by passing to formal Poisson structures modulo formal diffeomorphisms: a formal diffeomorphism is a map $\exp (X)$ with a formal vector field $X=\lambda X_{1}+\lambda^{2} X_{2}+\cdots \in \lambda \Gamma^{\infty}(T(T M))[[\lambda]]$ starting in order $\lambda$ to make the exponential series well-defined. The action of $\exp (X)$ on $\theta$ is the usual one.

In our case, we are interested in vertical formal Poisson structures and vertical formal diffeomorphisms, i.e., the vector field $X$ has to be vertical. On the star product side one considers equivalence classes of star products modulo equivalence transformations $S=\mathrm{Id}+\sum_{r=1}^{\infty} \lambda^{r} S_{r}$ where $S_{r}$ is a differential operator 
and $S$ acts on $\star$ by $f \star^{\prime} g=S^{-1}(S f \star S g)$. Again, in our case we are interested in vertical equivalence transformations, i.e., all the operators $S_{r}$ are vertical differential operators. Then one has the following statement [2, Appendix A]

Theorem 3. Let $\mathcal{U} \subseteq T M$ be an open neighbourhood of the zero section. Then

1. For every formal vertical Poisson structure $\theta$ with $\operatorname{supp} \theta \subseteq \mathcal{U}$ there exists a formal vertical star product $\star$ with cochains $C_{r}$ with $\operatorname{supp} C_{r} \subseteq \mathcal{U}$ for $r \geq 1$.

2. The vertical equivalence classes of vertical formal Poisson structures are in bijection to the vertical equivalence classes of vertical formal star products.

3. There exists a vertical formality map.

We do not state precisely what a formality is but refer to [2] where such a vertical formality is build out of Kontsevich's formality on $\mathbb{R}^{n}$ as in [6]. From this formality theorem the other two statements follow easily.

For the case of a $\mathcal{U}$-admissible Poisson structure $\theta$ with corresponding commuting vector fields $X_{i}$ as in (8) the existence of a star product quantizing $\theta$ is trivial: the explicit formula

$$
f \star g=\mu \circ \exp \left(\frac{\mathrm{i} \lambda}{2} \Theta^{k \ell} \mathcal{L}_{X_{k}} \otimes \mathcal{L}_{X_{\ell}}\right)(f \otimes g)
$$

does the job, where $\mu(f \otimes g)=f g$ denotes the pointwise product. This is the wellknown statement of Gerstenhaber on commuting derivations [4, Theorem 8]. The usual Weyl-Moyal star product on flat space is a particular case of Gerstenhaber's formula (10).

\section{Rieffel's Strict Deformation Quantization for $C^{*}$-Algebras}

In [11], Rieffel constructs a convergent noncommutative product for a dense subalgebra of a given Fréchet algebra $\mathcal{A}$. Further he proves, that the resulting noncommutative algebra is even a pre- $C^{*}$-algebra, if $\mathcal{A}$ is already a $C^{*}$-algebra. In this section we give a short survey on Rieffel's construction.

We consider a Fréchet algebra $\mathcal{A}$ with a strongly continuous action $\alpha$ of a vector space $V$ of dimension $d$. Moreover, $\alpha$ is required to be isometric, i.e., there exists a family $\mathfrak{P}$ of continuous seminorms defining the topology of $\mathcal{A}$ such that $p\left(\alpha_{v}(a)\right)=p(a)$ for all $p \in \mathfrak{P}, a \in \mathcal{A}$, and $v \in V$. In the case of a Banach algebra this reduces to the usual definition of isometric actions. 
The space of smooth vectors for the action $\alpha$ will be denoted by $\mathcal{A}^{\infty}$. It is well known that $\mathcal{A}^{\infty}$ carries a Fréchet topology. Moreover, it is dense in $\mathcal{A}$ with respect to the original Fréchet topology, see e.g., [13, Theorem A.1]. The action of $V$ on itself by translations induces an action $\tau$ of $V$ on $C_{u}^{0}(V, \mathcal{A})$.

We require now a scalar product on $V$ and choose a skew-symmetric operator $\Theta$ on $V$. Then

$$
a \bullet b=\iint \alpha_{\Theta u}(a) \alpha_{v}(b) \mathrm{e}^{2 \pi \mathrm{i} u \cdot v} \mathrm{~d} u \mathrm{~d} v
$$

is a noncommutative associative product for $\mathcal{A}^{\infty}$, called the deformed product (determined by $\alpha$ and $\Theta$ ), see [11, Definition 2.1, Theorem 2.14]. Here the integrals are understood in the sense of oscillatory integrals that are well-defined according to [11, Proposition 1.6]. We need the following [11, Corolary 2.13]

Proposition 4. Let $a \in \mathcal{A}^{\infty}$ be a fixed point for the action $\alpha$. Then for any $b \in \mathcal{A}^{\infty}$, we have $a \bullet b=a b$ and $b \bullet a=b a$.

Now, let $\mathcal{A}$ be a $C^{*}$-algebra. In order to define a $C^{*}$-norm for the deformed algebra $\left(\mathcal{A}^{\infty}, \bullet\right)$, one considers the space of all functions in $C_{u}^{0}(V, \mathcal{A})^{\infty}$ such that the product of their derivatives with any polynomial on $V$ is bounded. This space will be denoted by $\mathcal{S}^{\mathcal{A}}(V)$. On $\mathcal{S}^{\mathcal{A}}(V)$ Rieffel defines an $\mathcal{A}$-valued inner product

$$
\langle f, g\rangle=\int f(v)^{*} g(v) \mathrm{d} v
$$

together with a norm

$$
\|f\|=\|\langle f, f\rangle\|^{\frac{1}{2}} \text {. }
$$

Using the action $\tau$ of $V$ on the Fréchet algebra $C_{u}^{0}(V, \mathcal{A})^{\infty}$ one obtain a deformed product $\bullet$ for $C_{u}^{0}(V, \mathcal{A})^{\infty}$. Let $\mathrm{L}$ denote the left multiplications, i.e., the action of $C_{u}^{0}(V, \mathcal{A})^{\infty}$ on $\mathcal{S}^{\mathcal{A}}(V)$ using the deformed product

$$
\mathrm{L}_{F} g=F \bullet g \text {. }
$$

It turns out that the operator $\mathrm{L}_{f}: \mathcal{S}^{\mathcal{A}}(V) \longrightarrow \mathcal{S}^{\mathcal{A}}(V)$ is bounded and adjointable for $f \in \mathcal{S}^{\mathcal{A}}(V)$, see [11, Corolary 4.4]. Furthermore, this result also holds for the more general case $F \in C_{u}^{0}(V, \mathcal{A})^{\infty}$, see [11, Theorem 4.6].

For any $a \in \mathcal{A}$, Rieffel defines the function $\phi(a)$ on $V$ by

$$
\phi(a)(v)=\alpha_{v}(a) .
$$

Since $\alpha$ is isometric it turns out that $\phi(a) \in C_{u}^{0}(V, \mathcal{A})$. Moreover, the map $a \mapsto \phi(a)$ constitutes an equivariant ${ }^{*}$-homomorphism from $\mathcal{A}$ into $C_{u}^{0}(V, \mathcal{A})$. 
Thus it maps smooth vectors to smooth vectors, i.e., $\mathcal{A}^{\infty}$ into $C_{u}^{0}(V, \mathcal{A})^{\infty}$. It follows that it is a homomorphism for the corresponding deformed products [11, Proposition 2.10]. Therefore, $a \in \mathcal{A}^{\infty}$ determines a bounded operator on $\mathcal{S}^{\mathcal{A}}(V)$ by $\mathrm{L}_{\phi(a)}$. Thus one can pull-back the operator norm to $\mathcal{A}^{\infty}$ by

$$
\|a\|_{\Theta}=\left\|\mathrm{L}_{\phi(a)}\right\| \text {. }
$$

As the adjointable operators on a Hilbert module over a $C^{*}$-algebra form again a $C^{*}$-algebra [7, p. 8], it follows that $\|\cdot\|_{\Theta}$ fulfills the $C^{*}$-condition. After completion of $\left(\mathcal{A}^{\infty}, \bullet,\|\cdot\|_{\Theta}\right)$ one finally obtains a $C^{*}$-algebra. We refer to this $C^{*}$ topology as the deformed topology.

To conclude this section we recall the statement of [11, Theorem 5.7] that permits to obtain homomorphisms that are continuous with respect to the deformed $C^{*}$ topologies from certain continuous homomorphisms of the original undeformed $C^{*}$-algebras. Let $\alpha$ and $\beta$ be actions of $V$ on $C^{*}$-algebras $\mathcal{A}$ and $\mathcal{B}$, respectively. Moreover, let $\Psi: \mathcal{A} \longrightarrow \mathcal{B}$ be a ${ }^{*}$-homomorphism which is equivariant. Then the induced map $\Psi: \mathcal{A}^{\infty} \longrightarrow \mathcal{B}^{\infty}$ is a homomorphism of the respective deformed products which is continuous with respect to the deformed $C^{*}$-norms and hence extends to a continuous homomorphism for the respective completions. This result will be one of the key ingredients for the proof of an analogous statement in the case of pro- $C^{*}$-algebras, see Proposition 7.

\section{The Pro- $C^{*}$-Algebra Case}

In this section we will show that Rieffel's construction also works for pro- $C^{*}$ algebras and we will point out where some additional work was to be done.

Let $\mathcal{A}=\lim \mathcal{A}_{\lambda}$ be a pro- $C^{*}$-algebra, i.e., the inverse (or projective) limit of an inverse system of $C^{*}$-algebras $\mathcal{A}_{\lambda}$ in the category of topological ${ }^{*}$-algebras, see e.g. $[9,10]$. Recall that an inverse system of $C^{*}$-algebras consists of a directed set $\Lambda$, a $C^{*}$-algebra $\mathcal{A}_{\lambda}$ for each $\lambda \in \Lambda$, and ${ }^{*}$-homomorphisms $\pi_{\lambda, \rho}: \mathcal{A}_{\lambda} \longrightarrow \mathcal{A}_{\rho}$ for $\lambda \geq \rho$, satisfying the following conditions

$$
\pi_{\lambda, \lambda}=\operatorname{Id}_{\mathcal{A}_{\lambda}} \quad \text { and } \quad \pi_{\rho, \mu} \circ \pi_{\lambda, \rho}=\pi_{\lambda, \mu} \quad \text { for } \lambda \geq \rho \geq \mu .
$$

Then the inverse limit of $\left(\mathcal{A}_{\lambda}, \pi_{\lambda, \rho}\right)$ is a topological ${ }^{*}$-algebra $\mathcal{A}$ together with *-homomorphisms $\kappa_{\lambda}: \mathcal{A} \longrightarrow \mathcal{A}_{\lambda}$, such that

$$
\pi_{\lambda, \mu} \circ \kappa_{\lambda}=\kappa_{\mu}
$$

and satisfying the usual universal property as in [9]. Sometimes it will be convenient to identify an element $a \in \mathcal{A}$ with a coherent sequence $\left(a_{\lambda}\right) \in \prod_{\lambda \in \Lambda} \mathcal{A}_{\lambda}$ 
satisfying $\pi_{\lambda, \mu} a_{\lambda}=a_{\mu}$. The topology of $\mathcal{A}$ is determined by the set of all continuous $C^{*}$-seminorms on $\mathcal{A}$ denoted by $S(\mathcal{A})$, see [9, Proposition 1.1.1]. $S(\mathcal{A})$ is obviously a directed set by $q^{\prime} \geq q$ iff $q^{\prime}(a) \geq q(a)$ for all $a \in \mathcal{A}$. Defining $\mathcal{A}_{q}=\mathcal{A} / \operatorname{ker} q$ for $q \in S(\mathcal{A})$ one recovers the projective limit by [9, Proposition 1.1.1]

$$
\mathcal{A} \cong \lim _{\longleftarrow} \mathcal{A}_{q}
$$

An important example for a pro- $C^{*}$-algebra is the algebra of continuous functions $C^{0}(N)$ over a manifold $N$. It is the inverse limit of the inverse system of the $C^{*}$-algebras $\left(C^{0}(L),\|\cdot\|_{L}\right)$, where

$$
\|f\|_{L}=\sup _{x \in L}|f(x)|
$$

and $L$ runs through all compact subsets of $N$. Clearly, the compact sets are directed by $L^{\prime} \geq L$ iff $L^{\prime} \supseteq L$. The mappings $\pi_{L^{\prime}, L}: C^{0}\left(L^{\prime}\right) \longrightarrow C^{0}(L)$ for $L \subseteq L^{\prime}$ are the restriction maps and also the mappings $\kappa_{L}: C^{0}(N) \longrightarrow C^{0}(L)$ are given by restrictions

$$
\kappa_{L} f=i_{L}^{*} f
$$

Let $\alpha$ of $V$ be a strongly continuous action on a pro- $C^{*}$-algebra $\mathcal{A}$.

Definition 5. The action $\alpha$ is called cofinally isometric if there exists a cofinal subset $\Lambda \subseteq S(\mathcal{A})$ such that for all $q \in \Lambda$, all $a \in \mathcal{A}$, and all $v \in V$ we have

$$
q\left(\alpha_{v}(a)\right)=q(a)
$$

Given such a cofinally isometric and strongly continuous action $\alpha$ on $\mathcal{A}$, we obtain a deformed product $\bullet$ on the smooth vectors $\mathcal{A}^{\infty}$ by the general results of Section 4. Now we want to define corresponding $C^{*}$-seminorms for $\left(\mathcal{A}^{\infty}, \bullet\right)$ in order to obtain also a pro- $C^{*}$-algebraic deformation.

We can proceed analogously to the case of $C^{*}$-algebras up to the definition of the deformed norm as in (16). Then we have to use the following construction: a Hilbert module $\mathcal{E}$ over a pro- $C^{*}$-algebra $\mathcal{A}$ is defined as in the $C^{*}$-algebraic case, see [10], where completeness is now understood with respect to the seminorms $\|\xi\|_{q}=q(\langle\xi, \xi\rangle)^{\frac{1}{2}}$ where $\xi \in \mathcal{E}$ and $q \in S(\mathcal{A})$. Then $\mathcal{E}_{q}$ is defined to be the quotient $\mathcal{E} / \operatorname{ker}\|\cdot\|_{q}$. It turns out to be a Hilbert module over the $C^{*}$-algebra $\mathcal{A}_{q}$ in the usual sense. Hence the continuous adjointable operators $\mathfrak{B}\left(\mathcal{E}_{q}\right)$ on $\mathcal{E}_{q}$ are a $C^{*}$-algebra with respect to the usual operator norm. Given $q \in S(\mathcal{A})$ and $T \in \mathfrak{B}(\mathcal{E})$, one obtains $T_{q} \in \mathfrak{B}\left(\mathcal{E}_{q}\right)$ defined by $T_{q}[\xi]_{q}=[T \xi]_{q}$, where $[\xi]_{q} \in \mathcal{E}_{q}$ 
denotes the class of $\xi$. Then $\mathfrak{B}(\mathcal{E}) \ni T \mapsto T_{q} \in \mathfrak{B}\left(\mathcal{E}_{q}\right)$ is clearly an (algebraic) *-homomorphism whence

$$
\|T\|_{q}=\left\|T_{q}\right\|
$$

defines a $C^{*}$-seminorm for $\mathfrak{B}(\mathcal{E})$ for each $q \in S(\mathcal{A})$. We shall use this pro- $C^{*}$ topology which coincides with the one in [10]. It allows to define the pro- $C^{*}$ seminorms

$$
\|a\|_{\Theta, q}=\left\|\mathrm{L}_{\phi(a)}\right\|_{q}
$$

for $a \in\left(\mathcal{A}^{\infty}, \bullet\right)$ as in the $C^{*}$-algebraic case before. After completion we obtain a pro- $C^{*}$-algebra as wanted [5, Proposition A.4]

Proposition 6. For a pro- $C^{*}$-algebra $\mathcal{A}$ endowed with a strongly continuous and cofinally isometric action $\alpha$ of $V$ the deformed algebra $\left(\mathcal{A}^{\infty}, \bullet\right)$ of smooth vectors carries a system of $C^{*}$-seminorms defined by (21). The completion with respect to these seminorms yields a pro- $C^{*}$-algebra deforming $\mathcal{A}$.

As in the $C^{*}$-algebraic case the construction behaves well with respect to equivariant morphisms: let $\mathcal{A}$ and $\mathcal{B}$ be pro- $C^{*}$-algebras equipped with strongly continuous and cofinally isometric actions of $V$ and let $\Psi: \mathcal{A} \longrightarrow \mathcal{B}$ be an equivariant continuous ${ }^{*}$-homomorphism. Then we have [5, Proposition A.5]

Proposition 7. With these assumptions $\Psi:\left(\mathcal{A}^{\infty}, \bullet\right) \longrightarrow\left(\mathcal{B}^{\infty}, \bullet\right)$ is a continuous ${ }^{*}$-homomorphism with respect to the deformed pro- $C^{*}$-topologies.

\section{Strict Deformation Quantization for Actions with Compact Support}

In this section, we apply Rieffel's strict deformation quantization presented so far to construct a noncommutative product for the $C^{*}$-algebra of all bounded continuous functions $C_{b}^{0}(N)$ on a manifold $N$ and the pro- $C^{*}$-algebra of continuous functions $C^{0}(N)$ on $N$, respectively, provided we have a sufficiently nice action of $\mathbb{R}^{d}$. This will be used later for the locally noncommutative spacetime but may also be interesting for its own.

Let $K \subseteq N$ be a compact subset. We choose $d$ vector fields $X_{1}, \ldots, X_{d} \in$ $\Gamma^{\infty}(T N)$ with the following properties

1. $\operatorname{supp} X_{i} \subseteq K \subseteq N$ for $i=1, \ldots, d$

2. $\left[X_{i}, X_{j}\right]=0$ for $i, j=1, \ldots, d$. 
Clearly, there exist non-trivial choices of such vector fields. Since their supports are compact, their flows are complete. Moreover, their flows $\phi^{X_{1}}, \ldots, \phi^{X_{d}}$ determine an action of $\mathbb{R}^{d}$ on $C_{b}^{0}(N)$ by

$$
\alpha(v, f)=\alpha_{v}(f)=f \circ \phi_{v_{1}}^{X_{1}} \circ \cdots \circ \phi_{v_{d}}^{X_{d}} .
$$

Since the $X_{i}$ commute this indeed defines an action which turns out to be strongly continuous and obviously isometric with respect to the supremum norm on $C_{b}^{0}(N)$. Therefore it is possible to apply Rieffel's construction in this situation.

Definition 8. Let $\Theta$ be a linear and skew-symmetric operator on $\mathbb{R}^{d}$ with respect to the standard inner product. The noncommutative product $\bullet: C_{b}^{0}(N)^{\infty} \times$ $C_{b}^{0}(N)^{\infty} \longrightarrow C_{b}^{0}(N)^{\infty}$ is defined by

$$
f \bullet g=\iint \alpha_{\Theta u}(f) \alpha_{v}(g) \mathrm{e}^{2 \pi \mathrm{i} u \cdot v} \mathrm{~d} u \mathrm{~d} v .
$$

As $C_{b}^{0}(N)$ is a $C^{*}$-Algebra, the preceding results allow to define a $C^{*}$-norm $\|\cdot\|_{\Theta}$ on $C_{b}^{0}(N)^{\infty}$ such that the completion of $\left(C_{b}^{0}(N)^{\infty}, \bullet,\|\cdot\|_{\Theta}\right)$ becomes a $C^{*}$-algebra. Note that since the flows of $X_{1}, \ldots, X_{d}$ are smooth, we have $C_{0}^{\infty}(N) \subseteq C_{b}^{0}(N)^{\infty}$.

Since the action has compact support, the subalgebra of those functions in $C_{b}^{0}(N)^{\infty}$ with compact support $C_{b}^{0}(N)^{\infty} \cap C_{0}^{0}(N)$ remains a subalgebra with respect to the deformed product.

Proposition 9. The functions in $C_{b}^{0}(N)^{\infty}$ with compact support form a subalgebra with respect to the deformed product $\bullet$, i.e., for $f, g \in C_{b}^{0}(N)^{\infty} \cap C_{0}^{0}(N)$ we have $f \bullet g \in C_{b}^{0}(N)^{\infty} \cap C_{0}^{0}(N)$. More explicitly

$$
\operatorname{supp}(f \bullet g) \subset(\operatorname{supp} f \cap \operatorname{supp} g) \cup K .
$$

It is not difficult to see that the functions $f \in C_{b}^{0}(N)^{\infty}$ whose supports have an empty intersection with $K$ form a central *-ideal of $\left(C_{b}^{0}(N)^{\infty}, \bullet,\|\cdot\|_{\Theta}\right)$.

Proposition 10. For $f \in C_{b}^{0}(N)^{\infty}$ with supp $f \cap K=\emptyset$ we have

$$
f \bullet g=f g=g \bullet f \quad \text { for all } g \in C_{b}^{0}(N)^{\infty} .
$$

In particular, $\operatorname{supp}(f \bullet g) \cap K=\emptyset=\operatorname{supp}(g \bullet f) \cap K$.

The above construction for the $C^{*}$-algebra $C_{b}^{0}(N)$ is of course just an application of Rieffel's original construction. However, thanks to the support condition we can extend his result also to the following two situations: 
Remark 11. First, and this is still covered by Rieffel's general theory, we can use the $C^{*}$-algebra $C_{\infty}^{0}(N)$ of continuous functions vanishing at infinity instead of $C_{b}^{0}(N)$, i.e., the $C^{*}$-completion of $C_{0}^{0}(N)$. Then all results remain true for the corresponding deformation $\left(C_{\infty}^{0}(N)^{\infty}, \bullet,\|\cdot\|_{\Theta}\right)$ of $C_{\infty}^{0}(N)$.

Remark 12. Second, we can replace the bounded continuous functions by the pro- $C^{*}$-algebra $C^{0}(N)$ of all continuous functions. Here the property that the supports of the vector fields $X_{1}, \ldots, X_{d}$ are compact comes in crucially: indeed, first note that the action $\alpha$ on $C^{0}(N)$ is strongly continuous since on a compact subset a continuous function is uniformly continuous. Moreover, $\alpha$ is cofinally isometric as for each sufficiently large compactum $L \supseteq K$ we clearly have

$$
\|f\|_{L}=\left\|\alpha_{v}(f)\right\|_{L} \quad \text { for all } v \in \mathbb{R}^{d}
$$

for the sup-norm $\|\cdot\|_{L}$ over $L$. Thus the more general construction of a deformed pro- $C^{*}$-algebra can be carried through yielding a deformation $\bullet$ for $C^{0}(N)^{\infty}$. A straightforward verification shows that the assertions of the Proposition 9 and Proposition 10 literally hold true for $C^{0}(N)$ in the place of $C_{b}^{0}(N)$ as well.

\section{7. (Pro-) $C^{*}$-Algebraic Version of Locally Noncommutative Spacetimes}

This section will be devoted to the concrete construction of deformed products that incorporate the idea of a locally noncommutative spacetime in the framework of (pro-) $C^{*}$-algebras. As in the formal case, we will proceed in several steps: first we construct a suitable action of $\mathbb{R}^{d}$ on $T M$ for an arbitrary smooth manifold $M$ that induces a deformed product on $C^{0}(T M)^{\infty}$. This will require a more particular class of vertical Poisson structures. Then we use the exponential map to obtain induced products on $C^{0}(M \times M)^{\infty}$ and $C^{0}(M)^{\infty}$. The guiding principle of the respective construction clearly are the relations that are already known from the formal deformation quantization framework in Section 2. Furthermore, we will clarify the relations between the different products which algebraically are completely analogous to the formal case but include additional continuity properties. Clearly, all constructions will have their formulations for the $C^{*}$-algebraic cases of $C_{\infty}^{0}$ and $C_{b}^{0}$.

Let $\mathcal{U}$ denote an open neighbourhood of the zero section in $T M$ as in Section 2. Let $\theta$ be a $\mathcal{U}$-admissible vertical Poisson structure on $T M$ with its corresponding vertical action of $\mathbb{R}^{d}$ and (vertical) fundamental vector fields $X_{1}, \ldots, X_{d}$ as in Proposition 2. We denote the action of $\mathbb{R}^{d}$ on functions again by $\alpha$. Then the properties of the fundamental vector fields imply the following lemma, even 
though the action itself will not have compact support: the compactness in vertical directions is sufficient.

Lemma 13. The action $\alpha$ on $C^{0}(T M)$ is cofinally isometric.

Let $\bullet: C^{0}(T M)^{\infty} \times C^{0}(T M)^{\infty} \longrightarrow C^{0}(T M)^{\infty}$ be the corresponding noncommutative product defined by the oscillatory integral

$$
f \bullet g=\iint \alpha_{\Theta u}(f) \alpha_{v}(g) \mathrm{e}^{2 \pi \mathrm{i} u \cdot v} \mathrm{~d} u \mathrm{~d} v .
$$

Moreover, we denote the respective noncommutative products of $C_{b}^{0}(T M)^{\infty}$ and $C_{\infty}^{0}(T M)^{\infty}$ again by $\bullet$. Finally, we get noncommutative products for $C^{0}(\mathcal{U})^{\infty}$, $C_{b}^{0}(\mathcal{U})^{\infty}$, and $C_{\infty}^{0}(\mathcal{U})^{\infty}$ thanks to the support properties of the action $\alpha$. All these products are continuous for the deformed (pro-) $C^{*}$-topologies according to the results of the Section 4 and Section 5.

Next we reconsider the map $\Phi$ according to (1) that allows to push forward the vector fields $X_{i}$ to vector fields $\tilde{X}_{i} \in \Gamma^{\infty}(T(M \times M))$ by extending them to be 0 outside of $\mathcal{V}$. This way we obtain an action $\tilde{\alpha}$ of $\mathbb{R}^{d}$ on $C^{0}(M \times M)$ making $\Phi$ equivariant. Again, $\tilde{\alpha}$ is cofinally isometric. Thus the action gives rise to a deformed product $\tilde{\bullet}: C^{0}(M \times M)^{\infty} \times C^{0}(M \times M)^{\infty} \longrightarrow C^{0}(M \times M)^{\infty}$. Analogously to the case of $T M$ we also obtain deformed products for the spaces $C_{b}^{0}(M \times M)^{\infty}, C_{\infty}^{0}(M \times M)^{\infty}, C^{0}(\mathcal{V})^{\infty}, C_{b}^{0}(\mathcal{V})^{\infty}$, and $C_{\infty}^{0}(\mathcal{V})^{\infty}$ which are continuous for the corresponding deformed (pro-) $C^{*}$-topologies. The various products are related as follows, see [5, Proposition 4.2]

Proposition 14. The restriction map induces $a^{*}$-homomorphism $\left(C^{0}(T M)^{\infty}, \bullet\right)$ $\longrightarrow\left(C^{0}(\mathcal{U})^{\infty}, \bullet\right)$ which is continuous with respect to the deformed pro- $C^{*}$-topologies. The same statement holds for the case $C_{b}^{0}$ with respect to the deformed $C^{*}$-topologies. Moreover, the inclusion $\left(C_{\infty}^{0}(\mathcal{U})^{\infty}, \bullet\right) \longrightarrow\left(C_{\infty}^{0}(T M)^{\infty}, \bullet\right)$ is a continuous *-homomorphism with respect to the deformed $C^{*}$-topologies. Finally, the analogous results hold for $M \times M$ and $\mathcal{V}$ instead of $T M$ and $\mathcal{U}$.

Note that there is neither a direct relation between the classical algebras $C^{0}(T M)$ and $C^{0}(M \times M)$ nor the deformed algebras $\left(C^{0}(T M)^{\infty}, \bullet\right)$ and $\left(C^{0}(M \times M)^{\infty}, \tilde{\bullet}\right)$. Only when passing to the functions on $\mathcal{U}$ and $\mathcal{V}$, respectively, we obtain (see [5, Proposition 4.3])

Proposition 15. The pull-back map $\Phi^{*}: C^{0}(\mathcal{V}) \longrightarrow C^{0}(\mathcal{U})$ restricts to $a^{*}$ isomorphism

$$
\Phi^{*}:\left(C^{0}(\mathcal{V})^{\infty}, \tilde{\bullet}\right) \longrightarrow\left(C^{0}(\mathcal{U})^{\infty}, \bullet\right)
$$


which is continuous with respect to the deformed pro- $C^{*}$-topologies. It restricts to ${ }^{*}$-isomorphisms $\Phi^{*}:\left(C_{b}^{0}(\mathcal{V})^{\infty}, \tilde{\bullet}\right) \longrightarrow\left(C_{b}^{0}(\mathcal{U})^{\infty}, \bullet\right)$ and $\Phi^{*}:\left(C_{\infty}^{0}(\mathcal{V})^{\infty}, \tilde{\bullet}\right) \longrightarrow$ $\left(C_{\infty}^{0}(\mathcal{U})^{\infty}, \bullet\right)$, both continuous with respect to the $C^{*}$-topologies. In all cases, the inverse is given by $\Phi_{*}$.

In order to obtain the analogue $\tilde{\bullet}_{p}$ of $\tilde{\star}_{p}$ on the functions $C^{0}(M)^{\infty}$ on $M$ we have to proceed as follows:

First, we want to show that $\iota_{p}^{*}$ gives rise to a continuous homomorphism of the algebras $\left(C^{0}(T M)^{\infty}, \bullet\right)$ and $\left(C^{0}\left(T_{p} M\right)^{\infty}, \bullet p\right)$, where the latter product is obtained as follows: due to the verticality of the $X_{i}$ these vector fields restrict to $\iota_{p}$-related vector fields $X_{i}^{p} \in \Gamma^{\infty}\left(T\left(T_{p} M\right)\right)$. Consequently, also their flows are $\iota_{p}$-related, i.e., $\iota_{p}$ and hence also $\iota_{p}^{*}$ is equivariant. Using these flows we again get a strongly continuous and cofinally isometric action $\alpha^{p}$ of $\mathbb{R}^{d}$ on $C^{0}\left(T_{p} M\right)$, which can be used to define a deformed product $\bullet_{p}$ on $C^{0}\left(T_{p} M\right)^{\infty}$ by the usual integral formula. Again, $\bullet_{p}$ is defined as well for $C_{b}^{0}\left(T_{p} M\right)^{\infty}$ and $C_{\infty}^{0}\left(T_{p} M\right)^{\infty}$. We have [5, Proposition 4.4]

Proposition 16. The restriction $i_{p}^{*}: C^{0}(T M) \longrightarrow C^{0}\left(T_{p} M\right)$ induces a ${ }^{*}$-homomorphism

$$
i_{p}^{*}:\left(C^{0}(T M)^{\infty}, \bullet\right) \longrightarrow\left(C^{0}\left(T_{p} M\right)^{\infty}, \bullet_{p}\right)
$$

that is continuous with respect to the deformed pro- $C^{*}$-topologies. Moreover, the analogous statement holds for the $C^{*}$-algebraic cases $C_{b}^{0}$ and $C_{\infty}^{0}$.

Now we are prepared to turn to the second step of the construction of a product for functions on $M$ that is only noncommutative in a small neighbourhood of $p$. On $\mathcal{V}_{p}=\exp _{p}\left(\mathcal{U}_{p}\right)$ we can push forward $X_{i}^{p}$ to $\tilde{X}_{i}^{p}$ which extends to a smooth vector field on $M$ thanks to the support condition. In fact, $\operatorname{supp} \tilde{X}_{i}^{p} \subseteq \mathcal{V}_{p}$ is compact again. This way we obtain an action of $\mathbb{R}^{d}$ on $M$ making $\exp _{p}$ equivariant and hence have an action $\tilde{\alpha}_{p}$ on the continuous functions on $M$. Again we can apply Rieffel's construction to obtain a deformed product for the (pro-) $C^{*}$ algebras $C^{0}(M), C_{b}^{0}(M)$, and $C_{\infty}^{0}(M)$, which will be denoted by $\tilde{\bullet}_{p}$. One has the following properties [5, Proposition 4.5]

Proposition 17. Let $p \in M$.

1. $\bullet_{p}$ restricts from $T_{p} M$ to $\mathcal{U}_{p}$ and $\tilde{\boldsymbol{\bullet}}_{p}$ restricts from $M$ to $\mathcal{V}_{p}$, analogously to Proposition 14 
2. The pull-back $\exp _{p}^{*}: C^{0}\left(\mathcal{V}_{p}\right) \longrightarrow C^{0}\left(\mathcal{U}_{p}\right)$ induces $a^{*}$-isomorphism

$$
\exp _{p}^{*}:\left(C^{0}\left(\mathcal{V}_{p}\right)^{\infty}, \tilde{\bullet}_{p}\right) \longrightarrow\left(C^{0}\left(\mathcal{U}_{p}^{\infty}\right), \bullet_{p}\right)
$$

which is continuous with respect to the deformed pro- $C^{*}$-topologies

3. The analogous statements hold for the $C^{*}$-algebraic cases $C_{b}^{0}$ and $C_{\infty}^{0}$.

Note that all the above *-homomorphisms are also continuous with respect to the Fréchet topologies of the spaces of smooth vectors. This already follows from Rieffel's original construction.

With the hitherto results of this section we have found the (pro-) $C^{*}$-algebraic and hence non-perturbative counterparts to the constructions from [2] presented in Section 2, where formal star products are used instead. To make this relation more precise we consider a real non-negative parameter $h \in \mathbb{R}_{0}^{+}$and replace the constants $\Theta^{k \ell}$ by $h \Theta^{k \ell}$ in all the prevailing formulas. All the stated results remain literally the same except that all the noncommutative associative products now depend on the additional parameter $h$ which will be indicated by a subscript $h$. Then we find:

Proposition 18. For all $f, g \in C^{0}(T M)^{\infty}$ the asymptotic expansion with respect to the topology of $C^{0}(T M)^{\infty}$ for $h \rightarrow 0$ of the product $f \bullet_{h} g$ is given by the Gerstenhaber formula (10), i.e.,

$$
f \bullet_{h} g \sim_{h \rightarrow 0} \mu \circ \exp \left(\frac{\mathrm{i} \hbar}{2} \Theta^{k \ell} \mathcal{L}_{X_{k}} \otimes \mathcal{L}_{X_{\ell}}\right)(f \otimes g)
$$

where $\hbar=\frac{h}{2 \pi}$. This formula clearly has his equivalents for the other function spaces $C_{\infty}^{0}(T M)^{\infty}$ and $C_{b}^{0}(T M)^{\infty}$. The completely analogous statement holds for the products $\tilde{\bullet}_{h}, \bullet_{p_{h}}$ and $\tilde{\bullet}_{p_{h}}$, respectively, where the vector fields $X_{k}$ have to be replaced by $\tilde{X}_{k}, X_{k}^{p}$ and $\tilde{X}_{k}^{p}$, respectively.

Let us conclude with a small outlook and perspective:

In order to understand better the locally noncommutative spacetimes one should investigate the state spaces for the deformed algebras as explicitly as possible. This will be of major importance for the physical interpretation, in particular for the measurement of the (pseudo-)Riemannian structure on the deformed spacetime.

Moreover, again for physical applications it is necessary to treat $\theta$ not as a parameter but as a dynamical quantity itself. Thus it is interesting to investigate the 
dependence of the deformed products on $\theta$. Note that this goes (far!) beyond Rieffel's results on continuous fields [11, Chapter 9] as varying $\theta$ particularly implies to vary the action itself.

Finally, the results in $[1,3]$ suggest that one can now start to construct quantum field theories on locally noncommutative spacetimes. Here, one obvious conceptual difficulty is how to interpret quantum fields on $T M$ instead of quantum fields on $M$.

\section{Acknowledgements}

Stefan Waldmann would like to thank the organizers for a wonderful week in Bialowieza as well as the participants for useful comments and discussions.

\section{References}

[1] Bahns D., Doplicher S., Fredenhagen K. and Piacitelli G., Ultraviolet Finite Quantum Field Theory on Quantum Spacetime, Commun. Math. Phys. 237 (2003) 221-241.

[2] Bahns D. and Waldmann S., Locally Noncommutative Space-Times, Preprint math.QA/0607745 (2006) 28 pages. To appear in Rev. Math. Phys.

[3] Doplicher S., Fredenhagen K. and Roberts J., The Quantum Structure of Spacetime at the Planck Scale and Quantum Fields, Commun. Math. Phys. 172 (1995) 187-220.

[4] Gerstenhaber M., On the Deformation of Rings and Algebras III, Ann. Math. 88 (1968) 1-34.

[5] Heller J., Neumaier N. and Waldmann S., A $C^{*}$-Algebraic Model for Locally Noncommutative Spacetimes, Preprint math.QA/0609850 (2006) 13 pages.

[6] Kontsevich M., Deformation Quantization of Poisson manifolds, Lett. Math. Phys. 66 (2003) 157-216.

[7] Lance E., Hilbert $C^{*}$-modules. A Toolkit for Operator Algebraists, London Mathematical Society Lecture Note Series, vol. 210, Cambridge University Press, Cambridge, 1995.

[8] Liao Y. and Sibold K., Spectral Representation and Dispersion Relations in Field Theory on Noncommutative Space, Phys. Lett. B 549 (2002) 352-361.

[9] Phillips N., Inverse Limits of $C^{*}$-Algebras and Applications, In: Operator Algebras and Applications, vol. 1, D. Evans and M. Takesaki (Eds), London 
Mathematical Society Lecture Note Series, vol. 135, Cambridge University Press, Cambridge, 1988, pp 127-185.

[10] Phillips N., Inverse Limits of $C^{*}$-Algebras, J. Operator Theory 19 (1988) 159-195.

[11] Rieffel M., Deformation Quantization for Actions of $\mathbb{R}^{d}$, Mem. Amer. Math. Soc. 106 (1993) 93 pp.

[12] Rieffel M., On the Operator Algebra for the Space-Time Uncertainty Relations, In: Operator Algebras and Quantum Field Theory, Doplicher S., Longo R., Roberts J. and Zsido L. (Eds), International Press, Cambridge, MA, 1997, pp. 374-382.

[13] Schweitzer L., Dense m-Convex Fréchet Subalgebras of Operator Algebra Crossed Products by Lie Groups, Int. J. Math. 4 (1993) 601-673.

Jakob G. Heller

Fakultät für Mathematik und Physik

Physikalisches Institut

Hermann-Herder-Strasse 3

79104 Freiburg

GERMANY

E-mail address: Jakob.Heller@physik.uni-freiburg.de

Nikolai Neumaier

Fakultät für Mathematik und Physik

Physikalisches Institut

Hermann-Herder-Strasse 3

79104 Freiburg

GERMANY

E-mail address: Nikolai.Neumaier@physik.uni-freiburg.de

Stefan Waldmann

Fakultät für Mathematik und Physik

Physikalisches Institut

Hermann-Herder-Strasse 3

79104 Freiburg

GERMANY

E-mail address: Stefan.Waldmann@physik.uni-freiburg.de 